\title{
Artificial Intelligence (AI) and Digital Transformation in the Insurance Market: A Case Study Analysis of BGL Group
}

\author{
Christopher P. Holland \\ Loughborough University \\ Leicestershire \\ LE13 3TU, UK \\ c.p.holland@lboro.ac.uk
}

\begin{abstract}
Artificial Intelligence (AI) is transforming the insurance industry and there are many examples in the business press and at industry conferences. However, there is a paucity of detailed conceptual analysis and evaluation of AI technology that places AI in a strategic context and considers how different AI applications fit together and form a coherent picture. In this paper, a detailed case study of BGL group, a leading European insurance firm, is presented. A general business process model of insurance companies is used to structure the analysis. Five AI applications are described using an insurance firm-customer data flow diagram, which illustrates the marketing impact of AI technology and shows the nature of the business value creation process. The results are generalized into an AI customer lifecycle model, which has broad applicability to digital transformation projects. The likely future direction of AI in insurance is outlined and further research opportunities are identified.
\end{abstract}

\section{Introduction}

Digital transformation is a complex phenomenon, involving digital systems, business process redesign, novel business models, and changes to the structure and management of value chains [1]. The topic has received attention from venture capitalists [2], academic researchers [3] and consultants [4]. Artificial Intelligence (AI) is creating a new set of digital capabilities for business organizations and represents a new wave of digital technology because it offers the possibility of human-like capabilities, at scale, and across all functional areas of the business and the value chain, as a hugely powerful General-Purpose Technology (GPT), with a vast array of potential business applications, especially in digital marketing.

The insurance industry has lagged other markets because of the powerful position of incumbent insurance firms, combined with regulatory constraints, which have limited the effects of new entrants and market disruption. However, this has changed dramatically over the past few years, with a thriving, global InsurTech market, a willingness of consumers and regulators to adopt and embrace new technology and the pro-active strategies of incumbent firms to exploit AI and digital technologies to protect their market positions and launch novel services and business models [5].

Although there are many examples of AI insurance applications in the business press and in industry conference presentations [6], there are few detailed analyses of AI in insurance that take a holistic perspective and conceptualize how the wide range of new AI projects relate to each other, and to articulate their broader and longer-term implications for insurance firms and markets. In particular there is a dearth of detailed case studies, which are invaluable to explore new and emerging phenomena, because they allow the researcher to analyze the technology in situ and to make sense of rich data sources, including the knowledge and expertise of managers grappling with AI technology, organizational and strategic issues [7].

In this paper, a detailed case study of BGL's AI strategy is presented. BGL group is an interesting case study because it covers a broad range of insurance activities of insurance design, pricing, distribution, outsourcing and price comparison websites, in a single organization. It is also a digital leader and has already disrupted the insurance distribution with price comparison website technologies and owns the leading UK brand 'comparethemarket' and similar brands in several international markets. The case is analyzed using a combination of business process and customer lifecycle models that have transformed the electronic marketing of insurance services.

\section{Methodology}

The case study method has a long tradition in Information Systems (IS) research [8] and is attractive because it allows the researcher to be rigorous in their approach to exploring contemporary and relevant problems, including new and emerging digital technologies such as AI [9][10]. AI is not a discrete 


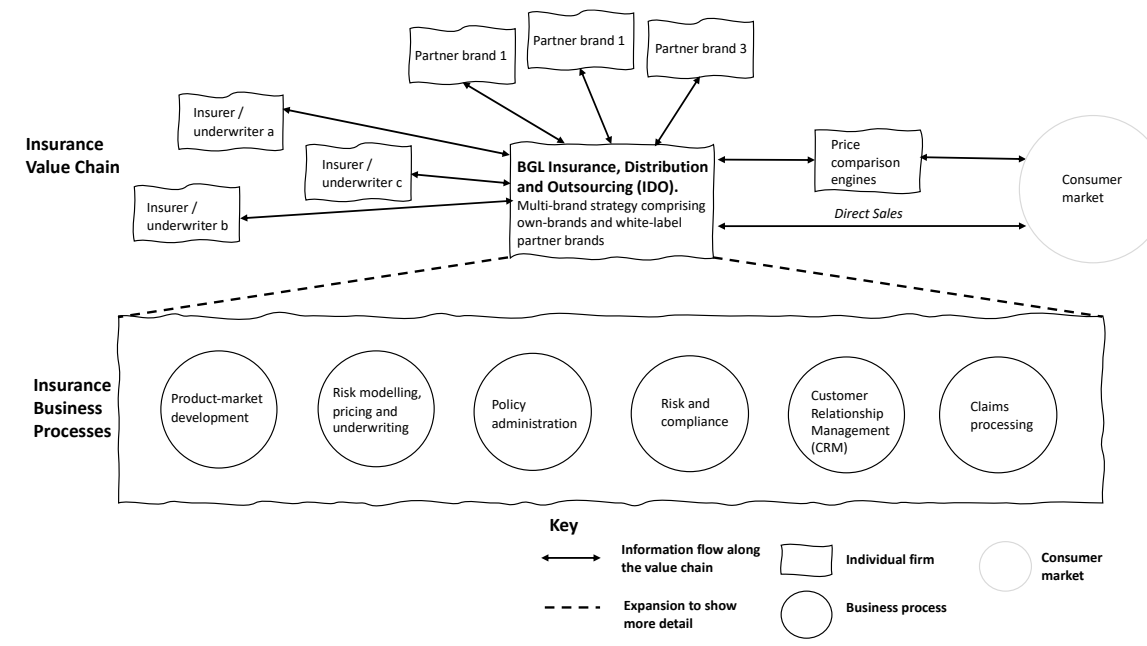

Figure 1. The insurance value chain and key business processes

technology but rather a qualitatively new approach to the design of algorithms that can change and adapt in the light of new data.

\subsection{Research framework}

Business model theory is a way of making sense of a business by analyzing its overall logic [11][12], and how the different business activities, or processes, fit together, e.g., how new customer acquisition operates to create new policies, which are then managed through eservice operations, and then renewed [13]. The concept of business models is especially useful to model the inter-relationships between digital technology and business processes [14].

The vast majority of AI systems insurance are narrow AI applications, i.e., they are used for a very specific, narrow business process, e.g., Robotic Process Automation (RPA) of legacy systems, smart policy management, new products based on behavioral risk in the health and automotive sectors, automated claims processing, chatbots and fraud detection. and underwriting. Each of these examples is a discrete, standalone AI application, and the challenge is to view them as part of a broader business logic that incorporates related digital systems, business processes and taken together, their combined effects on the overall business model [15].

\section{BGL case study}

BGL Group is a market leader in the distribution of insurance. It has a culture of digital leadership and has been a market disruptor in its use of price comparison websites in Europe, Australia, and South Africa, and adopts a digital first approach in its management of insurance services for partner organisations. The company has received numerous awards for its call centre operations, novel use of $\mathrm{AI}$ in fraud detection and the quality of its online customer journey design. BGL has a workforce of approximately 3,000 staff in Europe.

The company's reputation as a business disruptor, based on its novel use of technology to develop innovative business models, is an integral part of its culture and this approach is evident in its early adoption of Artificial Intelligence (AI). The group was established in 1992 as an insurance underwriter and has evolved through a combination of organic growth and a series of acquisitions to form two main divisions that operate autonomously from each other. The Price Comparison Division offers customers access to car, home insurance and a breadth of other products, e.g., life, travel, energy, pet insurance, utilities, and financial services. The Insurance, Distribution and Outsourcing (IDO) division sells motor, home and life personal insurance products through digital platforms and call centres. It has a multi-brand strategy consisting of its own brands and partnership brands, which are sold through affinity marketing agreements, i.e., white-label marketing agreements, where the partner brand outsources the product design and underwriting to BGL Group and its panel of insurers.

\subsection{Insurance value chain model}

A useful starting point to structure the analysis is the work by IBM [16], which identified six key business process areas: (1) product-market development; (2) risk modelling, pricing and underwriting; (3) insurance policy administration; (4) risk, compliance and regulation; (5) Customer Relationship Management (CRM); and (6) claims processing. This model has been adapted and extended into an insurance value chain framework, based on a synthesis of IBM's process model [16] and the insurance value chain concept [17] see Figure 1. 


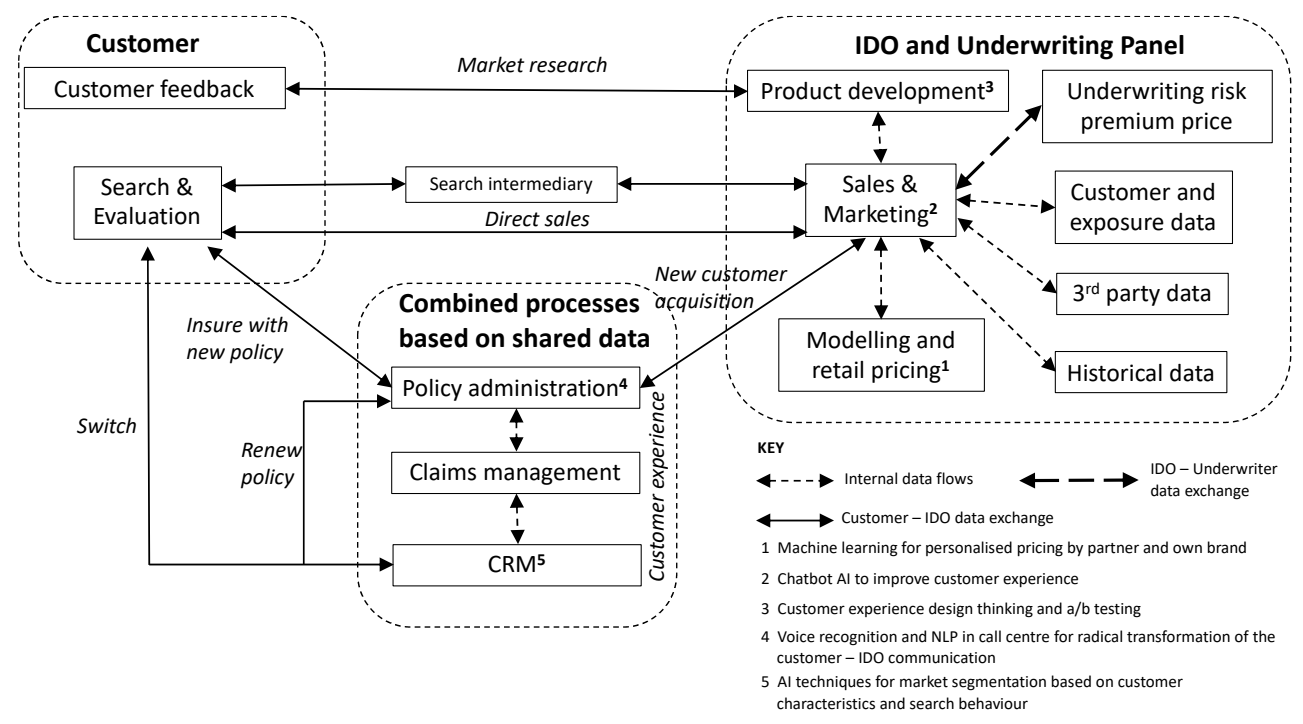

Figure 2. BGL's main AI applications, business processes and data flows

Starting with the customer market on the right-hand side of the diagram, consumers search for and evaluate competing insurance policies, either directly with an insurance firm, or via a search intermediary such as a price comparison website. Another channel option here would be through a personal broker which is the dominant channel in some European markets, e.g., Germany. BGL's Insurance, Distribution and Outsourcing (IDO) division links with 14 household partner brands to offer insurance services using the brand name of its partners, e.g., Marks \& Spencer and Virgin Money. The underwriting risk is then managed by BGL's panel of underwriters.

The marketing advantages to the partner brands are that they are able to extend the reach of their brand value into the insurance market, without the capital cost and market entry risks of developing an in-house insurance product. A variety of partnership arrangements is possible here, ranging from a simple affiliate marketing agreement that would involve a single payment to the partner brand for each sale through to more complex shared risk and reward agreements. To support the marketing of partner brand insurance products, shared marketing campaigns to existing partner brand customers also takes place.

\subsection{Key Insurance Business Processes}

A brief description of each set of key insurance processes is given. Product-market development is the creation of new insurance products that are targeted at specific market segments. Risk-modelling is used to inform pricing of insurance products, and the underwriting of the risk is the capital assigned in case a claim takes place. BGL's insurance partners are responsible for setting the risk price. As an intermediary, BGL is responsible for setting the 'street' price for each customer, which is the risk price plus margin. Policy administration is the management of the policy throughout its lifecycle from new customer acquisition through policy amendments, claims and termination of the policy. Efficient policy administration is important because it reduces the cost to serve customers and is an important service touchpoint with customers that influences their perception of the insurance firm and the likelihood to renew the policy and buy additional products.

The risk \& compliance process is about regulatory compliance, e.g., ensuring that the overall risk taken on by the insurance firm is within acceptable industry guidelines and that the company complies with industry rules and regulations, e.g., concerning timeliness of claims payments, insurance policy rules and appropriate use of customer data in marketing and fraud detection. Customer Relationship Management (CRM) is the ongoing marketing communication with customers throughout the product lifecycle. CRM has two crucial measures of success: (i) customer retention; and (ii) cross-selling of additional insurance products, e.g., a car insurance customer buying home insurance. Claims processing is a difficult administrative task to conduct effectively because the insurance firm must balance the need to provide a seamless, easy to use process for the customer that makes an appropriate payment in a timely manner, with the need to ensure that the claim is legitimate, and to manage the cost of the claims process itself. This is an analytical description of the processes, i.e., it describes them as individual, discrete processes. 


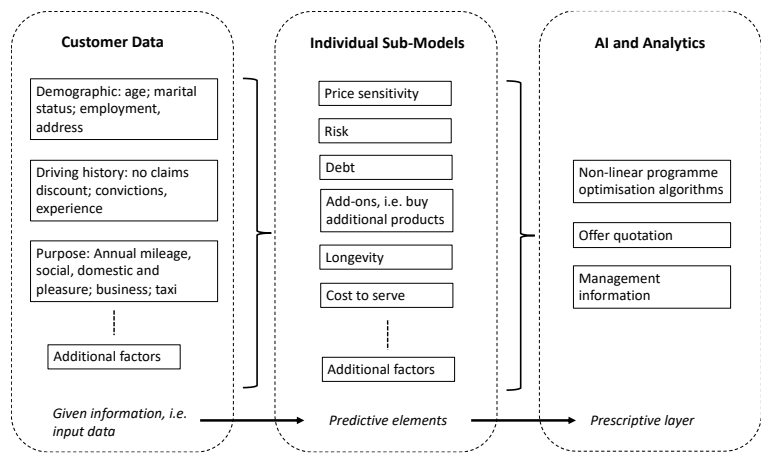

Figure 3. Pricing Model

There are two key research challenges here: (1) to describe how $\mathrm{AI}$ is affecting the design, operation, and performance of the individual processes; and (2) to explain how the processes fit together to create a higher order of business logic, i.e., to describe the AI-enabled business model. The approach taken is to map the individual AI applications and show how they are related to each other using data flows.

\subsection{AI applications mapped onto the customer lifecycle}

There are five main AI applications that relate to individual business processes or functions within the company. In Figure 2, each of the AI applications is mapped onto a data flow diagram of the company and its communication and data sharing for a typical customer lifecycle. Important features are shared processes and shared value creation. Customer value is derived from the customer's interactions with the insurance firm and the use of its products, i.e., value is co-created and also co-consumed by the insurance firm and its customers [18]. The insurance firm captures this value through the same set of communication processes. The application of Artificial Intelligence (AI) systems and more broadly digital technology is strategically important because it has the potential to significantly transform and improve value creation for both the insurance firm, e.g., improved data analytics, regulatory compliance, and better risk assessment; and the consumer, e.g., ease of use, less time in searching for and using insurance services, and data analytics for risk mitigation. Each of the AI systems is described below.

\subsubsection{Machine Learning for Pricing}

BGL's insurer partners are responsible for setting the risk price. BGL sets the retail price, which ensures customers are offered sustainable, competitive and fair prices.
"It is such a fast changing area that to have a specific plan of things to do seems a bit foolish, so our approach is to be very flexible and very agile in terms of the way we work and it is more around gathering together a group of very clever people, giving them very clear objectives, giving them very clear guidance as to what is acceptable or not - coming back to the regulatory side of things - and creating the environment where they are free to experiment in a controlled way and actually find what is good and bad." Thomas Bradley, Director of Pricing

This is an evolutionary strategy that adapts to newly available statistical techniques, AI algorithms, new data and technology improvements. Between 2010 - 2018, IDO used standard generalised linear models (GLMs) with a high degree of intervention by analysts using their experience and innate knowledge. GLMs are termed 'Generation 1' models. There has since been a constant iteration of techniques and approaches every 3-6 months to improve the modelling, and the firm is now in 'Generation 6' models that use AI optimisation techniques - see Figure 3.

The structure of the model is that there are three broad categories of information: (1) the given, or known information such as demographic information, exposure data and historical loss data; (2) the estimated information that use predictive behavioural models (termed sub-models; (3) the offer quotation that is calculated based on the optimisation of the individual sub-models in group 2. The range of individual elements within each category illustrate the information and analytical complexity of the online quotation process. The analytical complexity stems from the difficulty in estimating, or predicting expected values, in an accurate, robust and timely manner. The whole process of price production including calling our underwriters, third party data suppliers and running our own systems for up to 18 brands must happen in a few seconds following an online quotation request so time is of the essence.

The set of individual sub-models make predictions for specific elements of the model such as pricesensitivity of the customer, longevity, i.e. the expected lifetime of the customer, the number and frequency of mid-term adjustments to the policy, the overall expected cost to serve the customer and a range of additional factors. AI algorithms such as machine learning, combined with more traditional approaches, e.g., large, generalised linear regression models, are used at the individual sub-models to estimate individual values. For example, the expected longevity of the customer could be a function of (price, brand, age, type of use and annual mileage). 


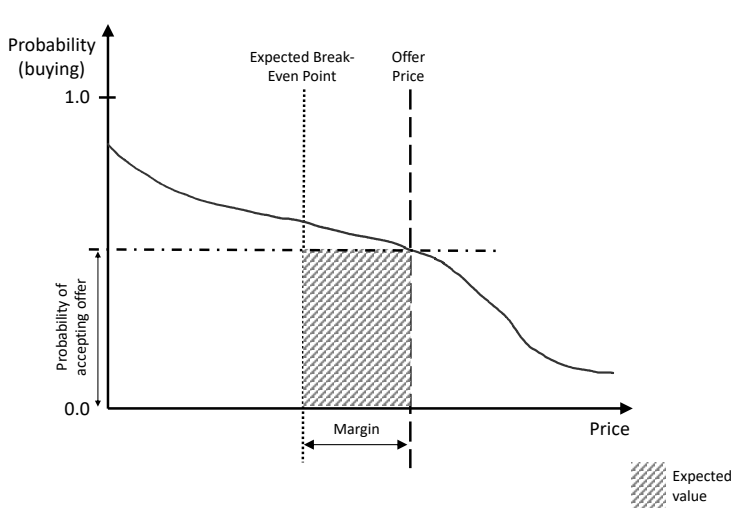

Figure 4. Price sensitivity curve

The crucial point about the modelling is that it is all data-driven and the models evolve in the light of new information. The models are developed in batch mode offline, but the actual quotation process for each potential individual customer is done in real-time.

"We build all these models offline, and we carry out this optimisation offline. We might build the model on a million customer quotes, we can then track to see who bought and at what price, how long they stayed with us, how much they cost to serve, etc. That's fine to run an optimisation algorithm offline in batch across a million customers to get an outcome, but the really clever thing is to get that optimisation algorithm to work in realtime, one quote at a time - that's where the Lagrangian mathematics comes in to translate that being done in batch mode to being able to work on individual quotes." Thomas Bradley, Director of Pricing

The results from the individual Machine Learning (ML) sub-models are combined with financial assumptions to create a function outlining the expected value at any given price for any given customer. The NPV functions are run across a representative sample of customer quotes. The optimisation algorithm then determines which price to offer to each customer in order to hit some global target or constraint while maximising the total expected value of the sample. As the optimisation can only affect the price, all nonpricing components are essentially static and for each given customer the only considerations are concerned with the demand and expected value at $0 \%$ mark up, price sensitivity, and how much those values change as the price is varied. An example of price sensitivity and expected value of the customer based on the estimated profit margin is shown in Figure 4.

On the vertical axis is the probability of buying the insurance product based on price sensitivity of a particular market segment. This is based on a Bayesian type approach that continually refines the model in the light of new information, i.e., accept / reject quotation offers for different product-market groups. The price sensitivity information is improved through a continuous process of testing and iteration of new market data.

"For a particular brand, we may set for a random $x \%$ of quotes, the set price recommended by the model. We'll then vary the price by $+/-y \%$ for the other $(1-x) \%$, i.e., for $(1-x) / 2 \%$ of quotes, use the set price recommended by the model $+y \%$, and for the remaining $(1-x) / 2 \%$ of quotes, use the set price recommended by model-y\%."

\section{Thomas Bradley, Director of Pricing.}

On the horizontal axis is price. Two prices are shown: (1) the offer price is the price that is quoted to the customer, which is the output of the model shown in Figure 4; (2) the expected break-even point is the insurance premium price that covers the expected costs of the insurance. The expected break-even point is a function of several main variables: predicted lifetime of the customer, customer propensity to buy additional products, and the cost to serve the customer, e.g., call centre and policy change costs. N.B. the individual variables have different effects on the expected breakeven point. The break-even point will increase as the customer risk increases. For customers who are likely to buy additional products at some time in the future, additional sales and profits reduce the break-even point. Some customers are predicted to have a higher cost to serve or are more likely to default on their insurance premium payments - both variables increase the breakeven point.

The price-sensitivity curve shows the probability of accepting the offer is a function of the quoted price however, the shape of the price sensitivity curve can vary greatly across different market segments and for different brands, which is why it is important to quote prices at the level of the individual. The value of the customer is equal to the offer price multiplied by the expected margin, i.e., offer price minus the break-even point. By expanding the component variables that estimate the break-even point, the expected value of an individual customer is a function of price, risk, price sensitivity, cost to serve, propensity to buy additional products plus other factors. This can be maximised using machine learning algorithms that are periodically evolved as new data becomes available through very large numbers of new online quotation outcomes and new customer and loss data.

In summary, customer data is assembled in standard templates and are the input into the sub-models that predict the variables that are used to calculate the expected value of the customer. The value function is then maximised to generate a quote for the insurance premium. The technical infrastructure is a pragmatic, 
flexible approach based on experimentation and innovation. A mix of proprietary software and internally developed machine learning algorithms are deployed on standard PC clusters that are managed in-house. The most important proprietary modelling software is called Earnix and is used to create and run the individual submodels that are shown in Figure 3. The quotation price is then derived by maximising the value function shown in Figure 4.

\subsubsection{Virtual Assistant (VA) chatbot AI}

For new and existing customers, a range of interactions between the customer and the insurance firm can take place, e.g., new customer acquisition, policy administration, claims processing, mid-term adjustments and renewal. IDO encourage customers to use the self-service channel, i.e. through a web portal, and take great care in designing specific customer journeys for each individual product such as car insurance or home insurance. A smart 'chatbot' that uses AI technology supports consumers in their use of the online channel by answering simple questions that then have the effect of encouraging consumers to continue using the online channel and to complete their transaction online, e.g. to change their address, add a new driver to a policy, change the vehicle or to renew the insurance. Customers can also simply phone the call centre directly. A schematic diagram of the overall system is shown in Figure 5.

Chatbot AI technology has two main purposes: to improve customer service so that customers are more likely to recommend IDO insurance products (both own-brand and partner brand products), and to reduce the cost-to-serve customers. Chatbot technology is an assistive tool that helps consumers navigate through common customer service requests, e.g., a renewal process or amendment to their policy. It replaces a human live chat with Natural Language Understanding (NLU) that is combined with detailed rule-based knowledge of the customer journey and insurance products. BGL's IDO division has developed its chatbot technology with Sabio, a technology company that specialises in using digital technology to enhance the customer experience. BGL's strategic use of key supplier partners such as Sabio is essential where expertise is in applying digital technology to enhance the customer experience in a contact centre environment is needed. The Virtual Assistant (VA) shown above for Budget Insurance automates some of the functions that are done using a traditional call centre. The emphasis at IDO is to encourage customers to use the online self-service channel because this is fully automated and therefore has a lower cost per transaction than the (human) call centre. However, many customers start to use the online channel and for whatever reason then switch to the call centre. VA technology is an attempt to overcome some of the inherent limitations of websites and to introduce a conversational style interface, which is preferred by many customers. The VA therefore increases the likelihood of customers remaining in the online channel. The operational benefits of the Budget VA technology have been to reduce phone and live chat by $1.1 \%$, to increase digital adoption by $10 \%$ and increase the online sales conversion by $0.5 \%$.

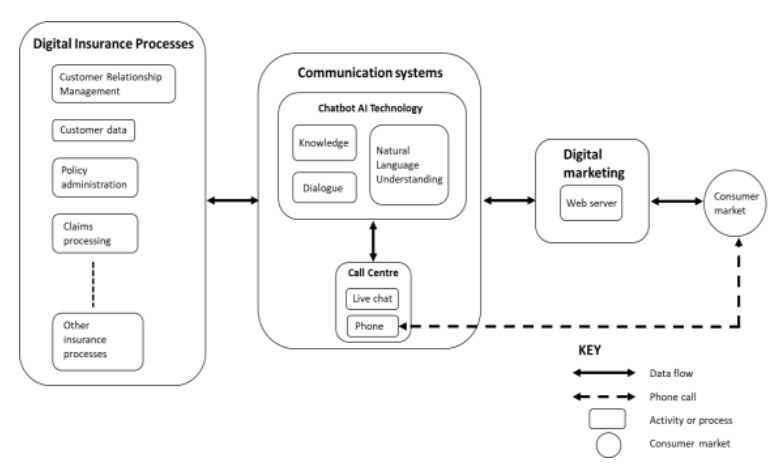

Figure 5. Schematic of Chatbot AI technology

\subsubsection{Customer experience design and $\mathbf{a} / \mathrm{b}$ testing}

A key feature of both the website and the Virtual Assistant (VA) technology is that they both rely on carefully planned sequences of activities. For each individual product there is a clearly defined customer journey to take a new customer from initial inquiry to customer information and authentication, to the purchase of the policy and the setting up of e-service. Once the customer has an active policy, there are then rules and associated online sequences of activities to manage the policy through e-service.

A website is a set of predefined links that works well on a specific customer journey from search through to buying, or for managing an e-service transaction. But what if customers wish to complete their task in a different way? A chatbot is inherently more flexible because it allows customers to ask questions and engage with the online system through a series of questions. A dedicated team analyse the customer experience through all digital channels and optimise the design from the perspective of the user, so that they are more likely to buy and renew their policies. A key element of this strategy is to use a combination of quantitative analysis and evaluation of users combined with qualitative research from focus groups and individual discussions with customers.

Quantitative testing of the existing website is based on the use of web analytics tools, which identify how users navigate through the website. This type of analysis 
yields important insights into longitudinal trends and online performance, but it does not tell the user experience managers why users are behaving in a particular way. Qualitative research methods such as focus groups are therefore used to complement the quantitative measures.

If the live statistics indicate a problem or opportunity for a redesign of an existing system, then the issue is explored using qualitative evaluation. In response to the first-hand insights generated, a new system is designed and evaluated using focus groups and then a rigorous $\mathrm{a} / \mathrm{b}$ testing approach. For example, if there are 3 or 4 competing ideas, each one is run with a large sample of customers, so that the team can get quantitative feedback quickly. More complex MultiVariate-Testing (MVT) of new customer journey designs is also carried out so that completely new designs with multiple changes/variables can be evaluated together. The results are then explored in a qualitative setting through either individual usability studies or focus groups of 12-20 users. These attempt to generate a detailed understanding of user behaviour. The design process philosophy is then to launch and test, iterate the design, optimise, and deploy, as quickly as possible. A potential weakness in a data driven approach is that it can lead to incremental changes and miss the 'breakthrough' moments. This means that creativity still has an important part to play in the design process.

"One of the reasons for conducting qualitative and quantitative research together, is that those breakthrough design moments are getting less and less frequent. Because there are so many competitors, so many websites, across all markets, if your design doesn't work with the right experience, people just leave. Trust in the website and the service of the company are so important. Bringing in the qualitative insight and adding it to the quant, means that we have a better chance of achieving those breakthrough design moments."

\section{Paul Humphrey, User Experience Research Manager, IDO, BGL Group.}

\subsubsection{Voice recognition}

The logic of voice technology is that voice will become an important channel in its own right as the technology improves and as consumers become more accustomed to using voice interfaces in other areas such as entertainment, ordering groceries, virtual personal assistants on mobile phones or managing home utilities. Voice technology is also related to the design of the websites because the idea of a voice conversation may also affect the design of the website.

At least in the experimental stages, the same kind of logic or brains behind the Virtual Assistant AI chatbot that is already used to manage virtual, typed conversations in the online channel will be used in the voice technology project. An unknown question here is whether customers using the voice channel will behave differently to when they use the online channel.

BGL's approach is to be an early adopter of this technology, experiment and learn, start with straightforward transactions such as common policy amendments.

"This is a nascent technology area, that's developing so quickly... especially, cloud computing, access to data, those are the two key ingredients for AI. They're both in abundance at relatively low cost. That wasn't the case 10 years ago, now it absolutely is the case. Another key point is that we're targeting simple calls that we feel are automatable - I've changed my car, I've changed my address, things like that. People still pick up the phone even though you could do this through self-service because they prefer that channel. The really important customer service angle is to do with customer experience - we would attempt for this to be zero wait $24 / 7$ service, which we do not provide today through our contact centres and no insurer does, as far as I know. No hold music, no waiting for an agent, it will always be available because it's not a real person. It'll be available around the clock."

\section{Stuart Walters, Chief Information Officer}

Digital voice is at the very start of the introductory stage of the product lifecycle, which means that it is very early in terms of technical development, user experience and sophistication. However, when it starts to improve, the market is likely to grow quickly and those companies that have experimented will be in an advantageous position to fully exploit the technology as it starts to mature, and as customers become accustomed to using it, and indeed, may come to expect it as an important e-service channel. BGL's strategy is to be in a strong position to grow with the market when it reaches the point of inflection, i.e., when it starts to grow quickly.

"When the voice channel takes off, we want to be there, whenever it happens, in three, four or five years' time, this will be a major new interface for customers."

\section{Stuart Walters, Chief Information Officer}

The voice calls will be guided calls and designed in a similar way to the flow of the insurer's website pages. The future limits of this technology are unknown, but the short-term goals are intended to be realistic and will be used to discover whether customers mind talking to a virtual assistant or robot, to see how customers 


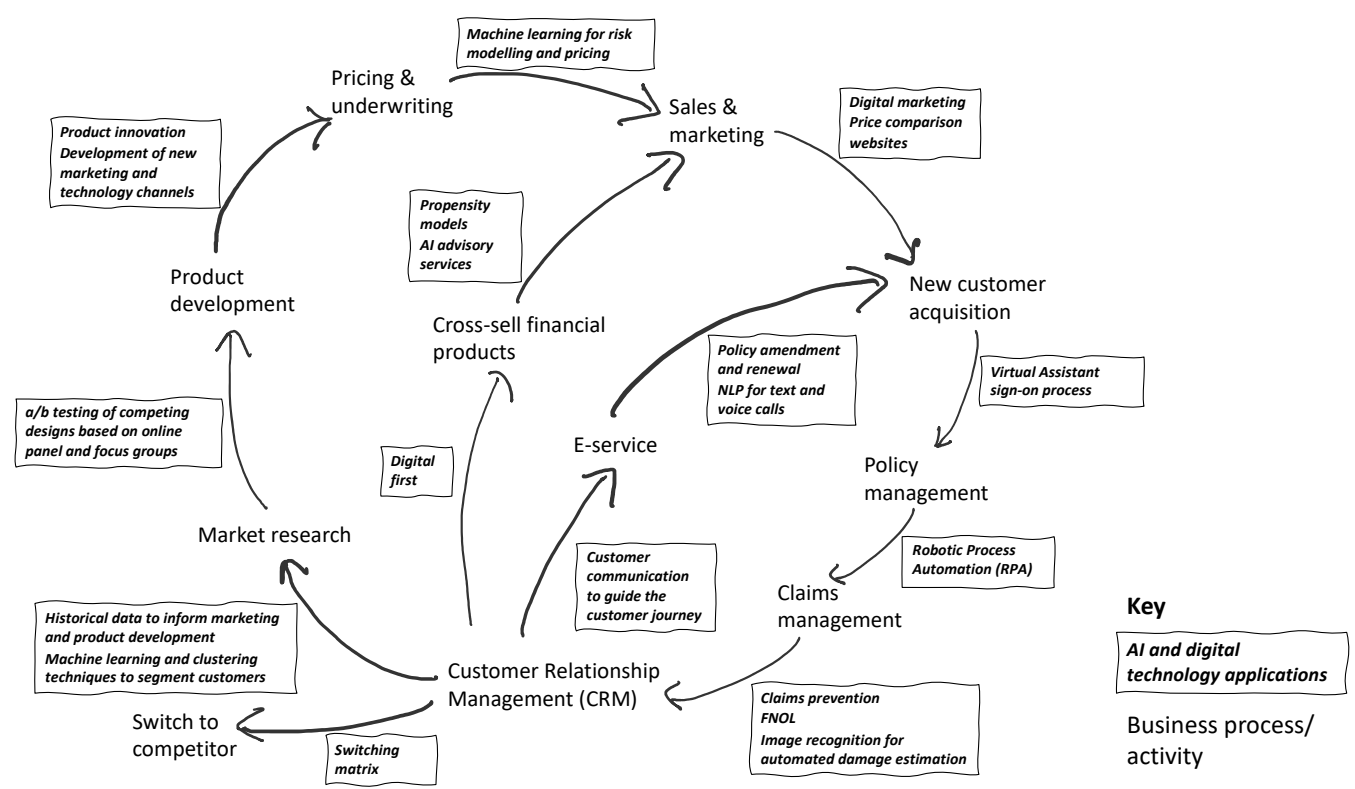

Figure 6. A dynamic model of AI applications mapped onto the online customer lifecycle

respond to pre-defined set of dialogues to emulate a natural conversational flow.

An important element in the implementation of a voice recognition system allied to a particular type of business application is access to relevant training data at scale, i.e., 100,000s of conversations from insurance call centre conversations, with different accents, issues to resolve and purposes to achieve. An incumbent such as BGL IDO therefore has an advantage in this respect over a new entrant because it has a vast library of conversation training data. An element of the training is the development of a comprehensive insurance ontology and detailed process maps and rules for different products, processes, and e-services. At this stage, BGL is experimenting with this highly advanced technology in partnership with Google Duplex and it is an early stage of evaluation.

\subsubsection{AI techniques for market segmentation}

The price comparison website 'comparethemarket.com' is the market leader for UK insurance price comparison websites. The meerkat characters are synonymous with the brand and used to promote three key areas: an innovative product that is

easy to use; rewards for usage such as film tickets and meal discounts; and industry-leading customer service. It offers price comparison in a range of vertical markets, including car, mortgages, broadband, credit cards, home insurance, life insurance, mobile phones, motorbike, pet, travel, and van insurance. The AI strategy is focusing on exploiting standardised Information Systems such as Salesforce CRM and combining them with AI systems such as machine learning for segmentation and estimating propensity to buy additional products based on historical transactions and new customer information that is shared through different marketing channels, i.e., call centre, mobile app, web and email.

The key point about the development of AI in this context is that AI is the tip of the iceberg in terms of related, or complementary investments in standard CRM technology, existing call centre technology, Virtual Assistant (AutoSergei) and mobile app technology. To trial a new AI application requires extensive training data and there is therefore a relatively high fixed cost to develop a new AI system and a significant barrier to entry for new entrants and smaller competitors, because they do not have access to a very large, historical database.

\section{Case discussion and proposed model}

The size and range of BGL's operations means that although it is a single case study, it is an effective sample to illustrate a wide variety of AI applications. The dynamic data flow diagram in Figure 2 illustrates the roles of the customer and the insurance firm separately and models the relationship as a set of shared business processes and data exchange. Value is created at the interfaces of the relationship, by the internal operations of the insurance firm, and the search strategies deployed by customers.

The five AI applications illustrate the range and variety of AI systems in insurance: new product development; pricing for individual customers; eservice chatbots; managing policy administration through sophisticated voice recognition and NLP; and creating 'smart' CRM by exploiting the wealth of customer-related data. These applications correspond 
closely to the customer journey, and a new model is proposed that uses the online customer journey as a framework to map all possible applications of AI in insurance for individual business processes - see Figure 6.

The plain text is the description of the business process/activity, and the boxes are AI applications, based on examples from BGL and AI use cases from the business press and management theory. Starting in the bottom left-hand corner with market segmentation, it moves through $\mathrm{a} / \mathrm{b}$ testing of new products, the development of new insurance services, e.g., behavioral insurance or pay per use, machine learning for modelling risk and pricing, the use of AI to apply advanced marketing techniques to exploit clickstream data, and for price/product feature comparison, and then into e-service using virtual assistant technology. For routine policy management, RPA technology has been widely used by other companies, and advanced claims management including image recognition has been documented. The last stage is CRM, which then divides into the customer switching to a competitor or retaining the customer with the possibility of cross-selling.

\section{Conclusions}

BGL has a culture of digital first and experimentation. New AI ideas are actively evaluated using prototypes, which results in a quick decisionmaking cycle to adopt and scale up or reject the idea. The digital infrastructure is kept up to date, which creates flexibility for new business approaches and novel technology by avoiding significant legacy system problems. A key element of the digital strategy is to use technology partners [19] and standard software in key areas such as CRM, data infrastructure, Virtual Assistants, call-centre technology, and machine learning algorithms. The internal digital expertise can therefore focus on integrating the technology into a cohesive platform that can exploit and develop its customer data and knowledge [20]. This is especially important in rapidly evolving areas such as voice technology where the technology giants such as Facebook, Google, Amazon, and Baidu have the necessary economic and technology scale to conduct research and develop cutting-edge products.

BGL has a wealth of customer data that is the raw material on which to develop and train AI systems to improve or solve specific business problems and processes. In competitive strategy terms, it is therefore not the AI itself per se that provides a competitive advantage, it's the AI system that combines the algorithm, the digital infrastructure and most importantly the big data that is used to train the machine learning so that the resulting AI system has superior performance over alternative approaches such as using people or standard management information systems.

Expanding beyond the findings of the individual case and relating these case results to the literature, some more general conclusions can be drawn. The detailed data flow model shown in Figure 2 is a powerful analytical method because it illustrates how the individual AI systems relate to each other and fit within the insurance firm-customer relationship. This context is essential to explore important higher-level phenomena, e.g., implementation, value creation and the crucial role of data throughout the business model.

The first wave of AI systems in insurance addressed narrow, specific problem tasks, e.g., Robotic Process Automation (RPA) to improve workflow in complex legacy environments, Virtual Assistant (VA) technology to improve customer experience, and machine learning to improve the modelling of complex optimisation problems in risk and pricing [5], [6]. These systems correspond to an individual business process or activity such as new customer acquisition, claims processing, fraud detection and policy management. The proposed model uses the customer lifecycle framework to map a comprehensive range of narrow AI applications that cover each stage of the customer lifecycle (Figure 6). This is a dynamic model and relates much more closely to the business model concept because it starts to explain the flow of data related to the logic of the business model. The customer lifecycle theory perspective also indicates the likely shape of future AI applications - this second wave of AI in insurance will be more ambitious in their business scope and in their level of algorithmic complexity, e.g., voice recognition, organization-wide AI regulation and integrated AI for the whole of the customer lifecycle.

The scale of big data generated from an insurance market leader's size and market position enable it to refine its value proposition, target customers more effectively and work successfully with partners, which in turn, generates additional data that can be used to improve and develop its AI algorithms, systems and decision-making processes [21], [22]. This means that market size and the novel use of big data is an advantage to incumbents and acts as a strategic barrier to entry that helps maintain the competitive positions of market leaders.

The online customer lifecycle model in figure 6 can be used to structure the digital transformation of firms in consumer markets by providing a customer lifecycle map of potential AI applications that shows how they relate to the customer journey. AI technology can be used to redesign and enhance internal business processes, customer-facing processes, and joint value creation at the interface of the relationship, and the model provides an overview and structure for the AI digital transformation strategy, and the use of data by 
the set of AI applications.

This analysis identifies further research opportunities. How can organisations such as banks, insurance and e-commerce firms integrate standalone, narrow AI applications to create a second generation of AI systems that are organization-wide in their scope and use of shared data? How should the responsible use of AI [23] be related to digital transformation? For example, the ethical use of customer data, avoidance of algorithmic bias, transparency of AI systems and explanations of automated decisions [24]. As AI systems grow in complexity and organizational reach, ethical factors must be considered in addition to technical, organizational, and strategic issues in AI digital transformation strategies.

\section{Acknowledgements}

This research is an output of the Technology and Next Generation Insurance Services TECHNGI project (www.techngi.uk) funded by Innovate UK and the Economic and Social Science Research Council (grant reference ES/S010416/1) as part of the $£ 20 \mathrm{M}$. Next Generation Services Research Challenge. The author would like to thank all the BGL staff who took part in the research, especially Stuart Walters and Jayne Lansdell, and Joseph Watson for research support in transcribing interviews. The views expressed in the paper are those of the author and do not necessarily represent those of BGL group. Any errors or omissions are the sole responsibility of the author.

\section{References}

[1] M. Ahmad, T. Botzkowski, C. Klötzer, and M. Papert, Behind the Blackbox of Digital Business Models. 2020.

[2] M. Andreessen, "Why Software Is Eating The World," Wall Street Journal, pp. 1-5, 2011.

[3] E. Brynjolfsson and A. Mcafee, The Second Machine Age: Work, Progress, and Prosperity in a Time of Brilliant Technologies. New York and London: W.W. Norton \& Company, 2016.

[4] P. Gandhi, S. Khanna, and S. Ramaswamy, "Which industries are the most digital," Harv. Bus. Rev., no. July, pp. 1-4, 2016.

[5] T. Catlin, J.-T. Lorenz, B. Münstermann, P. B. Olesen, and V. Ricciardi, "Insurtech - the threat that inspires," McKinsey Co., no. March, p. 12, 2017.

[6] J. Breeze, "Intelligent Automation," 2019. [Online]. Available:

https://www.intelligentinsurer.com/media/project_ii/do cument/1-james-breeze-axa-xl.pdf.

[7] R. K. Yin, Case Study Research and Applications: Design and Methods, 6th ed. SAGE Publications Inc., 2018.

[8] R. D. Galliers and F. F. Land, "Viewpoint: Choosing Appropriate Information Systems Research Methodologies," Commun. ACM, vol. 30, no. 11, pp.
901-902, Nov. 1987, doi: 10.1145/32206.315753.

[9] P. Darke, G. Shanks, and M. Broadbent, "Successfully completing case study research: Combining rigour, relevance and pragmatism," Inf. Syst. J., vol. 8, no. 4, pp. 273-289, 1998.

[10] E. Volmar and K. M. Eisenhardt, "Case study research: A state-of-the-art perspective," oxfordre.com. Oxford University Press, 2020.

[11] C. Baden-Fuller and M. S. Morgan, "Business models as models," Long Range Plann., vol. 43, no. 2-3, pp. 156-171, Apr. 2010.

[12] C. Zott and R. Amit, "Business model design and the performance of entrepreneurial firms," Organ. Sci., vol. 18, no. 2, pp. 181-199, Mar. 2007.

[13] A. Osterwalder and Y. Pigneur, Business Model Generation: A handbook for visionaries, game changers and challengers. Hoboken, New Jersey: John Wiley \& Sons, Inc., 2010.

[14] C. P. Holland and M. Gutiérrez-Leefmans, "A Taxonomy of SME E-Commerce Platforms Derived from a Market-Level Analysis," Int. J. Electron. Commer., vol. 22, no. 2, 2018.

[15] C. P. Holland, "Artificial Intelligence (AI) and Emerging Business Models in Insurance - Willis Towers Watson," Willis Research Network, 2019.

[16] IBM, "IBM Insurance Process and Service Models, General Information Manual," 2016. [Online]. Available: https://www.ibm.com/downloads/cas/VDZDBOGP

[17] M. Eling and M. Lehmann, "The Impact of Digitalization on the Insurance Value Chain and the Insurability of Risks," Geneva Pap. Risk Insur. Issues Pract., vol. 43, no. 3, pp. 359-396, Jul. 2018.

[18] S. L. Vargo and R. F. Lusch, "Service-dominant logic: Continuing the evolution," J. Acad. Mark. Sci., vol. 36, no. 1, pp. 1-10, 2008.

[19] C. P. Holland, "Influence of new business models and technology on technology strategy and partnerships," Routledge Companion to Manag. Digit. Outsourcing, pp. 41-59, Jul. 2020.

[20] F. Svahn, L. Mathiassen, and R. Lindgren, "Embracing digital innovation in incumbent firms: How Volvo Cars managed competing concerns," MIS Q. Manag. Inf. Syst., vol. 41, no. 1, pp. 239-253, 2017.

[21] R. Kimura, M. Reeves, and K. Whitaker, "The New Logic of Competition," BCG Henderson Inst., pp. 1-8, 2019, Accessed: Jun. 23, 2020. [Online]. Available: https://bcghendersoninstitute.com/winning-the-20s-thenew-logic-of-competition-7c1500c5a187.

[22] H. Chen, R. H. L. Chiang, and V. C. Storey, "Business intelligence and analytics: From big data to big impact," MIS Q. Manag. Inf. Syst., vol. 36, no. 4, pp. 1165-1188, 2012.

[23] B. Keller, "Promoting Responsible Artificial Intelligence in Insurance," Geneva Assoc. Insur. a Better World, no. January, pp. 1-4, 2020, [Online]. Available: www.genevaassociation.org.

[24] EIOPA, "Big data analytics in motor and health insurance," Frankfurt, 2019. [Online]. Available: https://register.eiopa.europa.eu/Publications/EIOPA_Bi gDataAnalytics_ThematicReview_April2019.pdf. 